\title{
ASTM Grain Size by EBSD - A New Standard
}

\author{
J. J. Friel, ${ }^{*}$ S. Wright,** and S. Sitzman*** \\ * Dept. of Earth and Environmental Science, Temple University, Philadelphia, PA 19122 \\ ** EDAX-TSL, Draper, UT 84020 \\ *** Oxford Instruments America, Concord, MA 01742
}

ASTM standards usually have their origin in a need by industry, government, or academic institutions. In the present case, there was a call from the aluminum industry to create a new standard practice to measure directly the size of space-filling grains in a microstructure. Grain size affects mechanical properties of materials, such as ductility, and ASTM standard E 112 has been used since 1955 to measure grain size using the light optical microscope (LOM). E 112 is widely used and works well but only if grain boundaries can be delineated by etching. In some materials, boundaries cannot always be revealed, so ASTM undertook to develop a practice for direct measurement by Electron BackScatter Diffraction (EBSD) in the SEM. Although ASTM has a subcommittee specifically on grain size (E 04.08), this work was assigned to subcommittee E 04.11 on X-ray and Electron Metallography, because the new document focuses on the EBSD technique and reports ASTM grain size in a manner consistent with E 112 and other existing grain size standards as listed in Table 1. ASTM G is recognized worldwide as a measure of grain size based on the number of grains/square inch. As such, the larger the number, $\mathrm{G}$, the smaller the grain size. $\mathrm{G}$ can be calculated from any of several stereological parameters such as: $\mathrm{N}_{\mathrm{L}}, \mathrm{N}_{\mathrm{A}}, \bar{A}$, and $\mathrm{P}_{\mathrm{L}}$, and $\mathrm{E}$ 2627 uses the average grain area, $\bar{A}$, to calculate $\mathrm{G}$ according to the following equation:

$G=(-3.3223 \cdot \log \bar{A})-2.995$ with $\bar{A}$ in $\mathrm{mm}^{2}$

After much discussion and several ballots, a document emerged from the ASTM process last year with consensus approval that can now be cited as E 2627, Standard Practice for Determining Average Grain Size Using Electron Backscatter Diffraction (EBSD) in Fully Recrystallized Polycrystalline Materials [2,3]. The new standard uses EBSD orientation maps to find grains, as shown in Fig. 1. It specifies the scan area and the minimum number of grains. Also specified are the limits of misorientation or tolerance angle to define grains, minimum points in the map needed to constitute a grain, and what to do with mis-indexed or non-indexed points. Table 2 shows the value of the parameters specified by the standard.

When compared with the LOM methods listed in Table 1, E 2627 provides a direct crystallographic measurement but is slower to use, especially when compared with a comparison chart method. In cases where etching does not reveal all the grain boundaries, image processing such as watershed segmentation or artificial intelligence may be used to complete the missing boundaries, as shown in Fig. 2. However, in cases where very few of the boundaries are revealed, grain determination by EBSD is by far the most useful and accurate technique. In addition to grain area, EBSD reveals texture, grain boundary character, other phases, and strain, all at spatial resolutions of 500nm or better. EBSD disadvantages include the need for an SEM/EBSD system and associated technical expertise, plus a required higher quality final polish than necessary for optical metallography.

To summarize the role of EBSD in grain sizing, orientation measurement is direct, and grains can be defined by tolerance angle. It achieves submicrometer resolution and can be implemented on most 
SEMs. E 2627 standardizes the acquisition conditions, and reports the widely-used parameter, ASTM G, based on average grain area. From the data distribution, the standard also calls for reporting the $95 \%$ confidence interval and percent relative accuracy. Other statistical parameters and a histogram of grain areas can be extracted from the data distribution and reported as desired.

\section{References}

[1] E 112 ASTM Annual Book of Standards, ASTM, West Conshohocken, PA

[2] E 2627 ASTM Annual Book of Standards, ASTM, West Conshohocken, PA

[3] S. Wright, et al., Workshop on Grain Size Determination According to ASTM E 112, E930, E1181, E1382, and other Methods, ASTM Int'1, San Antonio, 2010.

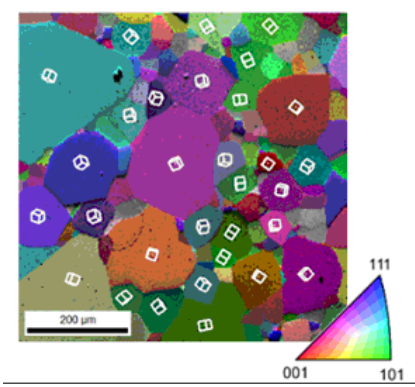

FIG. 1. Left - grain boundary map with color representing different orientations. The inverse pole figure legend is also shown. Right - pattern quality map with shading representing different orientations. The colors on the boundaries indicate the misorientation angle between two adjoining grains.

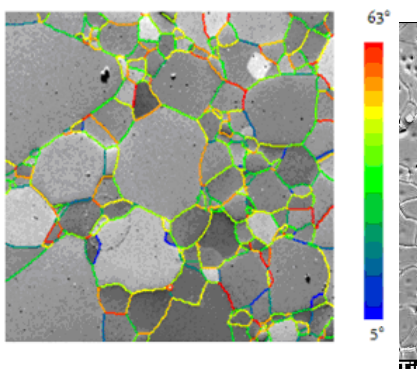

FIG. 2. Ceramic microstructure before $\&$ after grain boundary reconstruction by image processing using artificial intelligence.

TABLE 1. ASTM Grain Size Standards

\begin{tabular}{ll}
\hline ASTM Designation & Description of the Standard \\
\hline E 112 & ASTM G by comparison chart or stereological measurement \\
E 930 & ALA (as large as) grain size by comparison or measurement \\
E 1181 & Duplex grain size \\
E 1382 & ASTM G by automatic image analysis \\
E 2627 & ASTM G by EBSD \\
\hline
\end{tabular}

TABLE 2. Parameters Specified by E 2627

\begin{tabular}{ll}
\hline Grain misorientation & 5 degrees \\
Points per grain & $>100$ \\
Cleanup & $<10 \%$ of points modified \\
Scan area & $>50$ whole grains \\
Total no. of grains & $>500$ \\
\hline
\end{tabular}

